\title{
Oral midazolam for conscious sedation of children during minor procedures
}

\author{
F C Davies, $M$ Waters
}

\begin{abstract}
Objective-To compare the safety and efficacy of two doses of oral midazolam, and to assess the drug induced amnesia obtained, when used for conscious sedation of children undergoing minor procedures in the accident and emergency (A\&E) setting.

Methods-A two stage trial was completed: an initial prospective, double blinded, randomised trial comparing 0.2 mg/kg midazolam suspension with 0.5 $\mathrm{mg} / \mathrm{kg}$, followed by further data collection on the higher dose. Children whom staff and parents felt required sedation for accurate and humane completion of minor procedures were selected. Anxiety was measured using physiological parameters, a behavioural anxiety score, a parental visual analogue scale, and $a$ telephone questionnaire at 2-7 days after the procedure.
\end{abstract}

Results-Fifty patients in total were recruited. Randomisation between two doses ceased after 20 patients since staff, despite being "blinded", perceived there to be a wide variation in response to midazolam and attributed that to the difference in doses. On breaking the code these suspicions were partly supported. Due to reluctance to continue with the lower dose all children subsequently received $0.5 \mathrm{mg} / \mathrm{kg}$. At this higher dose oral midazolam had an onset of action of 15 minutes and was effective in $76 \%$ of children (as measured by anxiety score and/or subsequent amnesia). Amnesia was reported in $66 \%$ of children. There were no adverse side effects except paradoxical hyperagitation in three $(6 \%)$; this did not require any specific treatment. General anaesthesia was avoided in at least eight children in whom the procedure would not have been attempted without midazolam. Altogether $90 \%$ of parents said they would like it to be used again should similar circumstances arise.

Conclusions-At $0.5 \quad \mathrm{mg} / \mathrm{kg}$ oral midazolam appears safe and is effective in sedating most children for minor procedures. Its use should be considered by all $A \& E$ departments dealing with children.

(F Accid Emerg Med 1998;15:244-248)

Keywords: midazolam; conscious sedation; children; amnesia
Procedures which are performed relatively commonly in the accident and emergency (A\&E) department are often very frightening for children, and create a great deal of stress for parents and staff alike. Such procedures include wound repair, dressing of burns and fingertip injuries, foreign body removal, and eye examination. Current practice in this country and others usually begins with trying to cajole the child into cooperation. However, restraint techniques are needed for certain children, or for technically precise procedures. Under these circumstances the procedure is distressing for all concerned, and a variable degree of success is obtained. For this reason, selected procedures are often performed under general anaesthesia.

In recent years there has been a drive to find an effective, safe, short acting, non-injectable sedation agent for such circumstances. Oral midazolam has emerged as such an agent, and has the added benefit of inducing amnesia. The aim of this study was to assess the use of midazolam in this context in the UK, to help clarify the dose of midazolam required, add to the growing body of literature supporting its safety, and to assess the degree of amnesia resulting from its use.

\section{Methods}

A prospective, randomised, double blinded trial of a convenience sample of children was designed and ethics committee approval granted. Parents were given an information sheet and gave written, informed consent. Patients were excluded from the trial if they had received sedative medication in the previous 24 hours, had disabling respiratory disease, a coincidental head injury requiring hospital admission, known allergy to midazolam, or concurrent erythromycin treatment (due to drug interaction).

Patient selection was designed to resemble, as closely as possible, the wide range of situations encountered in daily practice in the A\&E department. Any child under 16 years old was considered, based on the perceived need for sedation by $A \& E$ staff. The need for sedation was determined by the degree of distress already present, or that anticipated considering the procedure about to be undertaken. This was determined by mutual agreement of the triage nurse, primary nurse, and middle grade or consultant level doctor, not by written selection criteria. Any type of minor procedure could be included, although longer procedures and those requiring accuracy were clearly more likely to be included. All patients received analgesia and/or local anaesthesia as necessary. 
Using information from previous studies patients were randomised to receive $0.2 \mathrm{mg} / \mathrm{kg}$ or $0.5 \mathrm{mg} / \mathrm{kg}$ of an oral midazolam solution. Two oral solutions of undisclosed strength were prepared by the pharmacy department who mixed intravenous midazolam $(10 \mathrm{mg} / 2$ $\mathrm{ml}$ ) with raspberry syrup to disguise its bitter flavour.

In accordance with the American Academy of Pediatrics guidelines ${ }^{1}$ full resuscitation facilities were available at all times and regular monitoring performed. Heart rate, respiratory rate, and oxygen saturations were documented at 10 minute intervals. Blood pressure was measured at initial assessment and at 10 minutes after administration of midazolam. Times were recorded for drug administration, readiness for the procedure, and fitness for discharge. Readiness for the procedure was judged by both doctor and nurse. Criteria used included cooperation with A\&E staff, signs of sleepiness, or a reduction in visible signs of distress such as crying. All procedures were performed in our "children's room" which contains toys and walls with murals. Distraction techniques were also employed, as they normally are with children in our department. The doctor performing the procedure was the doctor who initially assessed the child, or in some cases the maxillofacial on-call doctor.

The anxiety score was assessed using a previously validated scoring system based on observation of the child's behaviour. ${ }^{2}$ This scoring system is a hybrid of two anxiety scores (table 1). ${ }^{34}$ The score was documented at initial assessment, the time when the child was considered ready for the procedure, and during the procedure. For older children, the word "crying" was judged as synonymous with recurrent verbal indicators of distress.

The parents were asked to perform a visual analogue score at initial assessment of the injury and during the procedure. This consisted of a plastic sliding scale (made by Biomedical Engineering) with a line from "no pain" at one end, to "worst pain imaginable" at the other. It had a gradient of 0 to 100 on the flip side, not visible to the parents. Parents were asked to regard it as a scale of distress rather than pain. Patients were discharged when staff and parents agreed that they were alert and independently mobile (if they normally were).

A telephone questionnaire was performed at 2-7 days after the procedure. Parents were asked about the child's wellbeing, the incidence of side effects, and amnesia. A subjective opinion on the success of the midazolam was then sought. Parents were asked to choose from the phrases "very successful", "reasonably successful", "no difference", or "made things worse". They were also asked if they would like the same medicine to be used if the

Table 1 Observational anxiety score

\begin{tabular}{lll}
\hline Score & Behaviour before procedure & Behaviour during procedure \\
\hline 1 & Cooperative during examination & Cooperative or sleeping \\
2 & Crying only when wound is touched & Intermittent crying \\
3 & Crying during general examination & Continuous crying \\
4 & Uncontrolled crying & Uncontrolled crying \\
\hline
\end{tabular}

child attended the $\mathrm{A} \& \mathrm{E}$ department again under similar circumstances.

Successful anxiolysis was measured using physiological data (pulse, systolic blood pressure, and respiratory rate), the anxiety score, the visual analogue scale used by the parents, and the parental telephone questionnaire. Safety was evaluated by the above physiological data, including pulse oximetry. Amnesia was evaluated with the parental questionnaire.

Comparisons of differences between the two dosage groups were made at the $95 \%$ statistical significance level, unless stated otherwise. Comparisons of the data were made using the mean, median or proportions, as appropriate.

In order to increase the power of statistical analysis we sought to combine data from the two groups of children who received $0.5 \mathrm{mg} / \mathrm{kg}$ (those recruited during the randomisation phase and those afterwards). In order to ensure comparability of the groups, comparisons of baseline anxiety score, physiology, age, and final outcome were made. There were no significant differences found. The two groups have therefore been combined for all subsequent analyses.

\section{Results}

Fifty children were entered into the study. Telephone follow up questionnaires were completed on 42 children ( $84 \%)$. The median (range) age was 4 (1-13) years. The range of procedures performed included laceration repair $(56 \%)$, burn and fingertip dressings, foreign body removal, relocation of incisor teeth, application of a Thomas splint, reduction of a paraphimosis, and relocation of a dislocated thumb (table 2). All wound repairs in this study were with sutures, although Steristrips are commonly in use in the department.

In the first limb of the trial, children were randomised to receive a dose of $0.2 \mathrm{mg} / \mathrm{kg}$ or $0.5 \mathrm{mg} / \mathrm{kg}$ of oral midazolam. Staff and parents were blinded to the strengths of the two solutions. After 20 children had been recruited (nine to the $0.2 \mathrm{mg} / \mathrm{kg}$ solution and 11 to 0.5 $\mathrm{mg} / \mathrm{kg}$ ) the nursing staff noticed that in some children the midazolam appeared to have little or no effect. They suspected that the lower dose was less effective. Due to reluctance to continue with the lower dose we decided to continue to collect data using only the 0.5 $\mathrm{mg} / \mathrm{kg}$ solution. Therefore nine children received $0.2 \mathrm{mg} / \mathrm{kg}$ and 41 children ultimately received $0.5 \mathrm{mg} / \mathrm{kg}$.

Table 2 Procedures performed under sedation with oral midazolam

\begin{tabular}{ll}
\hline Procedure & No \\
\hline Laceration of face, pinna, or head & 18 \\
Other laceration & 9 \\
Removal of foreign body in hand or foot & 6 \\
Repair of crush injury to fingertip & 5 \\
Burn dressing & 2 \\
Earring removal & 2 \\
Replacing avulsed incisors & 2 \\
Cleaning/exploration of hand wounds & 1 \\
Reduction of dislocated thumb & 1 \\
Reduction of paraphimosis & 1 \\
Removal of zip from foreskin & 1 \\
Removal of foreign body from vagina & 1 \\
Application of Thomas splint & 1 \\
\hline
\end{tabular}


We tested the hypothesis that the nursing staff were correct in perceiving the lower dose to be less effective. On breaking the code their impression was supported by the parental questionnaire, although not by significant differences in anxiety scores, physiological data, or the parental visual analogue anxiety scale. When questioned, one of eight parents in the $0.2 \mathrm{mg} / \mathrm{kg}$ solution group rated the overall success of the medication as "very good", compared with 17 of 34 parents $(50 \%)$ in the $0.5 \mathrm{mg} / \mathrm{kg}$ group. This is a difference in proportions of $37.5 \%$ (95\% confidence interval 9 to 66). Overall 24 of the 34 parents replying in the higher dose group $(71 \%)$ rated the efficacy as either "very good" or "reasonably good".

An anxiety score of 1 or 2 was deemed as "effective sedation". At initial assessment a score of 1 or 2 was present in five of nine $(55 \%)$ in the lower dose group and 24 of $41(58 \%)$ in the higher dose group. These were the children in whom midazolam was given on the basis of the likely reaction once starting the procedure. After administration of midazolam a score of 1 or 2 was achieved in seven of the nine lower dose children (78\%) and 37 of the 41 given the higher dose $(90 \%)$. During the procedure the relative proportions were four of nine $(44 \%)$ and 25 of $41(61 \%)$ respectively. These differences are not statistically significant at the $95 \%$ level. The parental visual analogue anxiety score did not yield statistically significant results. Data for this part were frequently left incomplete.

The time from administration of midazolam to readiness for procedure was 15 minutes for $0.2 \mathrm{mg} / \mathrm{kg}$ (range 10-35) and 15 minutes for $0.5 \mathrm{mg} / \mathrm{kg}$ (range 5-55). The times to discharge were 49 minutes (range 35-50) and 60 minutes (range 30-100) respectively.

There were no clinically significant changes in heart rate, blood pressure, respiratory rate, or oxygen saturations in any child. The lowest recorded respiratory rate was $12 /$ minute and the lowest oxygen saturation was $91 \%$. There were no adverse effects documented in the $A \& E$ department or reported at follow up except for paradoxical hyperagitation in three cases $(6 \%)$. This is a recognised side effect of midazolam. All three cases occurred with the higher dose and none in the lower dose patients but this difference was not statistically significant.

There was no difference in achieving amnesia between the two dosage groups. From the 42 completed follow up questionnaires, 27 $(66 \%)$ of children had lost some memory of their attendance in A\&E. Parents were asked if the child had recalled any part of the procedure and $18(44 \%)$ replied no. Altogether $22 \%$ had some memory of the event, but had not expressed any negative feelings about it.

Thirty seven parents $(90 \%)$ said they would like the same medicine to be used again if similar circumstances arose.

After the 20th patient we added the subjective question "would this procedure have been attempted only under general anaesthesia had midazolam not been available?" to the assess- ment sheet. The answer clearly required the observer to use their previous experience of similar injuries and their likelihood of successful completion without sedation. In the 27 procedures for which the question was answered, 10 were answered yes. Only two of these subsequently required general anaesthesia after failing to achieve success with midazolam, thus probably "preventing" eight hospital admissions for a general anaesthetic.

There was no difference in the effectiveness of midazolam in relation to the child's age or in relation to procedures which involved suturing. However, when we compared procedures performed on the face (including pinna) with other procedures, we found that the anxiety score increased significantly during the procedure in 14 of 22 children requiring facial procedures compared with six out of 28 in the non-facial group, a difference of $42 \%$ (95\% confidence interval 17 to $67, \mathrm{p}=0.05$ ).

\section{Discussion}

In recent years there has been increasing interest in the sedation of children about to undergo distressing therapeutic procedures. Much of the published literature relates to premedication of children for operations. Such literature cannot necessarily be applied to the sedation of children presenting to the $\mathrm{A} \& \mathrm{E}$ department immediately after an injury. A useful recent summary of the experience relating to $A \& E$ practice was written in $1994 . .^{5}$ Clearly our study, along with those before it, is limited by the variation between different children, different injuries and circumstances, and the subjective nature of deeming sedation to be successful.

Currently in the UK reliance is often placed on restraint techniques or oral morphine solution if pain is the predominant problem. Morphine remains a useful drug but is a weak anxiolytic agent and causes nausea. Over the last few years a number of sedation or analgesic sedative agents have been tried with varying degrees of success, mainly in the United States. Most have been limited by one or more factors. Clearly the ideal sedation agent should not require injection since the fear of needles adds greatly to the anxiety associated with the procedure. Nitrous oxide is commonly found in $A \& E$ departments but its administration for long procedures necessitates continuous inhalation at a concentration of at least $30 \%$ by face mask. ${ }^{6}$ This may not be easy to achieve safely or reliably. Fentanyl lollipops were found to achieve adequate sedation and analgesia, but caused a high incidence of unacceptable side effects, namely vomiting (up to $47 \%$ ) and pruritus (up to $67 \%) .^{7}$ Oral ketamine solution has been used successfully with only minor side effects and few reports of emergence phenomena in those under 10 years old. However its onset of action is up to 45 minutes and discharge from the $A \& E$ department may be delayed for around 104 minutes (range 75-215). ${ }^{8}$ An intranasal combination of sufentanil and midazolam looks promising ${ }^{9}$ but sufentanil is not available in this country at present. 
Oral midazolam, a benzodiazepine, has been used with success in $A \& E$ since $1990^{210^{11}}$ and in a much larger number of trials as a premedicant. In these studies it fulfilled many criteria for an "ideal" sedation agent, with a rapid onset of action (around 12 minutes) and absence of serious side effects. The rectal ${ }^{12}$ and intranasal ${ }^{13}$ routes were found to have little practical benefit in terms of rapidity of onset of action, were less acceptable routes for the patient and caused local irritation. Midazolam is more potent when used by these routes and has been recorded to cause apnoea. ${ }^{14}$

We chose to use the oral route of administration and after considering the published literature we decided that it was, by now, unethical to compare midazolam with placebo, since it is known to be effective. We then sought to address some questions which remain outstanding. These include assessment of its efficacy for injuries other than lacerations, assessing the incidence of amnesia, establishing the appropriate dose, and testing its application in this context outside the United States. The doses of $0.2 \mathrm{mg} / \mathrm{kg}$ and $0.5 \mathrm{mg} / \mathrm{kg}$ were chosen on the basis of previous papers, ${ }^{21011}$ although higher doses have been used for premedication. ${ }^{15}$

Our methodology could be criticised on the following bases: firstly the subjective judgment about which children should receive sedation. This was difficult to avoid given the wide variation in children's behaviour and injury patterns. Secondly, for possible interobserver differences in assessment. However permitting only a single observer to run the study would have resulted in a much prolonged study period and in denial of midazolam to many children. Thirdly, the decision to abandon the lower dose after the 20th patient is also open to criticism. Due to the small numbers who received $0.2 \mathrm{mg} / \mathrm{kg}$ this left us with no firm conclusion regarding the best dose. In practice, however, it was impossible to continue with a dose in which staff had lost faith, despite being "blinded". Staff may simply have been observing the wide variability of response of patients to all benzodiazepines. The decision not to rerandomise to a dose higher than $0.5 \mathrm{mg} / \mathrm{kg}$ was made because of concerns about possible over-sedation in a busy district general hospital. Finally, with such a wide age range and variety of different procedures, analysis of results is clearly rather subjective.

No clear conclusions can be drawn about the $0.2 \mathrm{mg} / \mathrm{kg}$ dose on the basis of this study. However, we have shown that a dose of $0.5 \mathrm{mg} / \mathrm{kg}$ is safe and effective in producing anxiolysis and amnesia for a wide range of $A \& E$ procedures. Further studies are needed to clarify whether the optimum dose of midazolam for anxiolysis could be higher, without compromising its safety or side effect profile. The appropriate dose may need to vary with the procedure performed. We found that children remained more anxious in procedures involving the face or head (the majority of which were lacerations). Children find these procedures very threatening and it is perhaps not surprising that their fear was great enough to overcome the effect of the midazolam. Many comments on the assessment sheets related to distress during injection of local anaesthetic. Warming lignocaine to body temperature and adding alkali to reduce its acidity may reduce this pain.

Higher doses of midazolam may be associated with a greater incidence of side effects such as paradoxical hyperagitation. ${ }^{15} \mathrm{~A}$ varying incidence of hyperagitation has been previously reported, from $0 \%{ }^{9}$ to $6 \% .{ }^{12}$ We observed many children becoming "chatterboxes" but regarded only three as truly agitated $(6 \%)$. No specific treatment was required. All three had received the higher dose, although this could not achieve statistically significance because of the small numbers. The lowest recorded oxygen saturation was $91 \%$. It is not clear if this may have been due to technical difficulties. The minimum respiratory rate recorded was 12 (two patients, a 2 year old with minimum oxygen saturation of $95 \%$ and a 9 year old).

The anxiety score which we used has been used in several studies and is simple to apply to all children, irrespective of their verbal skills. We encountered some difficulties in using it as a tool for measuring the effectiveness of sedation. After an initial reduction in anxiety score after receiving midazolam, the score was frequently highest during the procedure. In addition, some children who were undistressed at initial assessment received sedation because staff predicted that they were likely to encounter difficulties during the procedure. In both situations this resulted in an increase in the anxiety score. However previous studies using a placebo group for comparison have confirmed that this increase in anxiety is reduced. To completely obtund the response to a procedure it may be necessary to sedate a child to a level which is potentially unsafe in the $A \& E$ setting. Furthermore the visual analogue sliding scale proved to be a difficult conceptual tool for parents. They found the concept of measuring distress as a linear scale difficult. Data were often missing or were at odds with comments documented on the forms.

The median time from administration of midazolam to the time when ready for the procedure was 15 minutes which is comparable with previous studies and with the information on the peak absorption of oral midazolam. ${ }^{14}$ The wide range documented (up to 55 minutes) probably reflects both the predictable occasions when staff are distracted by other things in a busy department and, in some cases, time spent waiting for an effect which was not going to happen. No children were observed to achieve greater sedation by waiting more than 15 minutes. Likewise the variation in time to discharge (30-100 minutes) probably reflects departmental factors, given the pharmacological profile of midazolam.

No previous studies have examined the amnesic effects of midazolam, possibly because of lack of follow up information. We found an incidence of $66 \%$ partial amnesia and $44 \%$ for the whole procedure, although this was judged by the parents taking their child's verbal skills and age into account. This benefit was 
extremely popular and led parents to say they would use it again even in those cases where the sedation appeared inadequate. They felt that bad memories were upsetting to the child, led to a phobia of hospitals and doctors, and made subsequent removal of sutures or changes of dressings a much more traumatic event. An overwhelming number $(90 \%)$ of parents would like it to be used again if similar circumstances arose. Explanations given were because of its amnesic effect, and because they were grateful for any attempt made to calm the child.

Finally, as well as providing more humane conditions for procedures, sedation may allow a better technical result. Furthermore, in eight of 10 cases where the question was included, the senior doctor involved felt that using sedation had obviated the need for general anaesthesia. Although the judgment was subjective, being based on previous experience, this potentially holds many benefits for both the child and the hospital.

\section{Summary}

In conclusion, our study supports previous studies in showing that oral midazolam, while not a panacea, is a useful drug for conscious sedation of children in the A\&E setting. At a dose of $0.5 \mathrm{mg} / \mathrm{kg}$ it provides at least reasonable sedation and/or amnesia for the majority of children ( $76 \%)$. Sedation appears to be less successful for procedures on the face. Measuring the success of sedation is difficult in objective terms, however it should now be considered unethical to compare midazolam with placebo in future studies.

Midazolam has an onset of action of $15 \mathrm{~min}$ utes and appears safe. There are as yet no reported cases of clinically significant respiratory depression at this dose. Unpleasant side effects are few (hyperagitation in 6\%), although many children become talkative. At least partial amnesia for the experience is induced in $66 \%$. This is perceived as a great benefit from the parents' points of view, and may reduce fear of subsequent contact with medical services. Lastly, its use in $A \& E$ may obviate the need for general anaesthesia in some cases.

Further studies are required to establish which is the optimal dose without compromis- ing its safety profile or increasing the incidence of paradoxical hyperagitation. It may be preferable to combine midazolam with other agents such as those related to fentanyl, particularly where analgesia is required. Further research will help us understand the appropriate role of oral midazolam in the repertoire of drugs and other techniques (such as play therapy and hypnosis) which we can use to reduce distress for children attending the $\mathrm{A} \& \mathrm{E}$ department.

With thanks to Dr P Williamson for her statistical advice, and to Dr E Fantom and Ms Stephanie Edwards for their help with data collection.

1 Committee on Drugs, American Academy of Pediatrics. Guidelines for monitoring and management of pediatric patients during and after sedation for diagnostic and therapeutic procedures. Pediatrics 1992;89:1110-15.

2 Hennes HM, Wagner V, Bonadio WA, et al. The effect of oral midazolam on anxiety of preschool children during oral midazolam on anxiety of preschool children

3 Herbertt R, Michaelinnees J. Familiarization and preparatory information in the reduction of anxiety in child denta patients. Journal of Dentistry for Children 1989;JulyAugust:319-22.

4 Venham L, Murry P, Gaulin-Kremer E. Personality factors affecting the preschool child's response to dental stress. J Dent Res 1979;58:2046-51.

5 Sacchetti A, Schafermeyer R, Gerardi M, et al, on behalf of the Pediatric Committee of the American College of Emergency Physicians. Pediatric analgesia and sedation. Ann Emerg Med 1994;23:237-50

6 Gamis AS, Knapp JF, Glenski JA. Nitrous oxide analgesia in a pediatric emergency department. Ann Emerg Med 1989, 18:177-81

7 Schutzmann SA, Burg J, Liebelt E, et al. Oral transmucosal fentanyl citrate for premedication of children undergoing laceration repair. Ann Emerg Med 1994;24:1059-64.

8 Qureshi FA, Mellis PT, McFadden MA. Efficacy of ora ketamine for providing sedation and analgesia to children requiring laceration repair. Pediatr Emerg Care 1995;11 93-7.

9 Bates BA, Schutzmann SA, Fleisher GR. A comparison of intranasal sufentanil and midazolam to intramuscular
meperidine, promethazine and chlorpromazine for conscious sedation in children. Ann Emerg Med 1994;24:64651 .

10 Taiwo B, Flowers M, Zoltie N. Reducing children's fear when undergoing painful procedures. Arch Emerg Med 1992;9:306-9.

11 Fatovich DM, Jacobs IG. A randomized, controlled trial of oral midazolam and buffered lidocaine for suturing lacerations in children (the SLIC trial). Ann Emerg Med 1995;25:209-14.

12 Shane SA, Fuchs SM, Khine H. Efficacy of recta midazolam for the sedation of preschool children undergoing laceration repair. Ann Emerg Med 1994;24:1065-73.

13 Connors K, Terndrup TE. Nasal versus oral midazolam for sedation of anxious children undergoing laceration repair Ann Emerg Med 1994;24:1074-9.

14 Malinovsky JM, Populaire C, Cozian A, et al. Premedication with midazolam in children. Effect of intranasal, rectal and oral routes on plasma midazolam concentrations. Anaesthesia 1995;50:351-4.

15 McMillan CO, Spahr-Schopfer IA, Sikich N, et al. Premedication of children with oral midazolam. Can J Anaesth 1992;39:545-50. 